2 Bourtourline-Young HJ, Smith CA. Respiration of full term and premature infants. Am $\mathcal{F}$ Dis Child 1950;80:753-63.

3 Seguin JH, Donovan EF, Kotagal HR. Respiratory timing in newborns with respiratory distress syndrome. Pediatr Res 1985;19:1515A

4 Hird M, Greenough A. Inflation time in mechanical ventilation of preterm neonates. Eur f Pediatr 1991;150:440-3.

5 South $M$. Observations of spontaneous respiration in ven- tilated babies. London: University of London, 1987 (MD thesis.)

6 Harrison VC, Heese $\mathrm{H}$ de V, Klein $M$. The significance of grunting in hyaline membrane disease. Pediatrics $1968 ; 41$ : 549-59.

7 Remmers JE, Bartlett D. Reflex control of expiratory airflow and duration. F Appl Physiol 1977;42:80-8.

\title{
Intraventricular haemorrhage after aspiration of ventricular reservoirs
}

\author{
N E Moghal, M W Quinn, M I Levene, J W L Puntis
}

\begin{abstract}
A previously unrecognised complication of aspirating ventricular reservoirs is described. Four infants developed fresh bleeding into the cerebrospinal fluid after reservoir taps; ultrasound confirmed intraventricular blood clot in one case. The technique for aspirating the reservoir may have an important bearing on the incidence of this complication.
\end{abstract}

The use of ventricular reservoirs in the management of the newborn infant with posthaemorrhagic hydrocephalus was first described in $1980 .{ }^{1}$ It has since been shown to be an effective method for 'buying time'. It allows time to stabilise the infant, prevents multiple lumbar punctures and ventricular taps, allows more aggressive management of raised intracranial pressure, and enables clearance from the cerebrospinal fluid (CSF) of blood and protein before insertion of a ventriculoperitoneal shunt. In addition, the incidence of shunt infection is significantly reduced when reservoirs are used initially with later shunting as compared with early primary insertion of a shunt. ${ }^{2}$ We review our experience of 10 cases who had reservoirs inserted over a three year period and describe a previously unrecognised complication-late intraventricular haemorrhage. of Paediatrics and Child Health, Peter Congdon Newborn Unit, Clarendon Wing, General Infirmary at Leeds, Leeds LS2 9NS N E Moghal M W Quinn $M$ I Levene J W L Puntis Correspondence to: Professor Levene.

Accepted 1 October 1991

(Arch Dis Child 1992;67:448-9)
University Department

\section{Patients and methods}

Between January 1988 and December 1990, 10 infants had reservoirs inserted at the Leeds General Infirmary for posthaemorrhagic hydrocephalus. Eight of the infants were ventilated for hyaline membrane disease and all suffered at least a grade II intraventricular haemorrhage in the first week of life. This was followed by progressive ventricular dilatation. Indications for inserting a reservoir were an occipitofrontal
Details of CSF removal

\begin{tabular}{|c|c|c|c|}
\hline & Gaskill et al ${ }^{3}$ & $\begin{array}{l}\text { Leonhardt } \\
\text { et al }\end{array}$ & $\begin{array}{l}\text { Leeds General } \\
\text { Infirmary }\end{array}$ \\
\hline $\begin{array}{l}\text { Median (range) volume removed (ml) at each tap } \\
\text { Median (range) tap frequency/day } \\
\text { Needle gauge used }\end{array}$ & $\begin{array}{l}*(5-15) \\
2 \text { (up to } 3) \\
27\end{array}$ & $\begin{array}{l}6(1-15) \\
2(1-4) \\
25-27\end{array}$ & $\begin{array}{l}11(1-36) \\
1(0 \cdot 5-3)\end{array}$ \\
\hline
\end{tabular}

${ }^{*}$ Median not stated. head circumference which was larger than the 97th centile, difficulty with lumbar punctures and/or symptoms of raised intracranial pressure. The reservoir was inserted in the parietooccipital region with the tip lying in the occipital horns of the lateral ventricles and the dome lying subcutaneously.

Subsequently, the reservoir was tapped sufficiently often to control excessive head growth or symptoms of raised intracranial pressure (most commonly apnoea and or bradycardia). The CSF was removed through a 23 gauge butterfly needle usually by free drainage. Occasionally gentle suction was applied by a syringe when CSF flow was sluggish. All patients had regular cranial ultrasound examinations and the CSF sent for microscopy and culture. Shunt insertion was performed when the CSF became clear of red blood cells and the protein concentration was less than $2 \mathrm{~g} / \mathrm{l}$.

\section{Results}

The median (range) weight of the infants treated with a reservoir was $1100 \mathrm{~g} \mathrm{(675-3560}$ g). The gestation ranged from 23 to 41 weeks. The reservoirs were inserted at 6 to 68 days postnatal age (median 27 days).

There were no reservoir infections. Two infants became hyponatraemic, probably due to CSF removal as their serum sodium was normal before this removal; this was corrected with sodium supplements in the feeds. No infant became hypoalbuminaemic. One reservoir became blocked and required revision: no specific cause was identified but infection was excluded.

There were four cases of late intraventricular haemorrhage after reservoir insertion. These episodes occurred at 13,17, 24, and 33 days after insertion. The CSF had become clear just before these bleeds. Fresh blood suddenly appeared in the CSF and red cell counts rose to between $10^{3} / 1$ and $10^{5} / 1$. In one case a new blood clot was seen on cranial ultrasound scan at the time of the haemorrhage. This bleed occurred into a porencephalic cyst on the opposite side to the reservoir (fig). Clotting studies at the time of the haemorrhage were normal.

The method and amount of cerebrospinal fluid removed is compared in the table with 

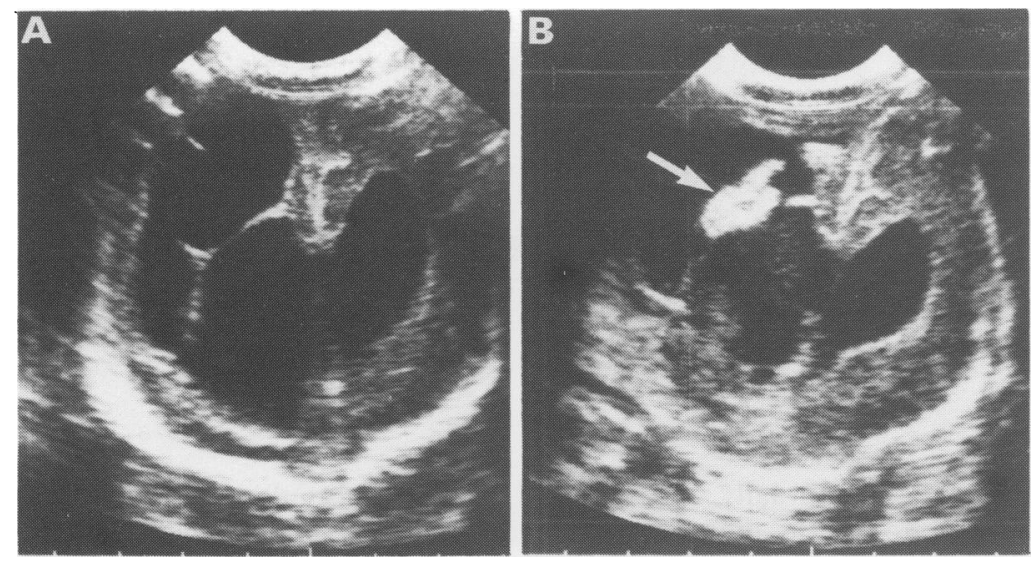

Coronal ultrasound scans showing $(A)$ large right sided porencephalic cyst and dilated ventricles before the haemorrhage and $(B)$ echodensity (arrowed) associated with haemorrhage in the region of the porencephalic cyst.

previous series of Gaskill $e t a l^{3}$ and Leonhard $e t$ $a l .{ }^{4}$ In those who had a late haemorrhage, the method of removing the CSF was analysed in more detail. There was no significant change in the volume and frequency of taps just before the haemorrhage. Those who had a haemorrhage had a similar volume and frequency of taps as those who did not. In the one case where an ultrasound scan confirmed the bleed, suction was used to remove CSF just before the bleed.

There were no symptoms in any of the patients at the time of the haemorrhage, but there was a delay in insertion of the ventriculoperitoneal shunt in those who suffered a haemorrhage (median age of insertion 80 days) compared with those who did not (median 20 days).

\section{Discussion}

Late haemorrhage as a complication during reservoir management of posthaemorrhagic hydrocephalus has not previously been reported. Although not associated with any symptoms, the four affected patients had a delay in conversion of the reservoirs to ventriculoperitoneal shunts. The possibility of such a complication was highlighted in a commentry by Bannister on the paper by Leonhardt et al. ${ }^{4}$ She emphasised that the removal of CSF via a reservoir is not physiological because it does not allow a smooth transition of intracranial pressure. Significant pressure differentials may be produced in the brain leading to rupture of friable blood vessels. The method of removing the CSF will have an important influence on the magnitude of these pressure differentials.

In comparison with Leonhardt et al and Gaskill et al (table), we removed on average more CSF less frequently with a larger gauge needle and, on occasion, with the application of gentle suction. These factors may be important and possibly explain our observation of secondary intraventricular haemorrhage. In order to minimise the pressure shifts within the brain, we recommend more frequent taps (at least daily), removing smaller volumes $(<10 \mathrm{ml})$ and using smaller gauge needles $(25 \mathrm{G}, 27 \mathrm{G})$. The CSF should be allowed to drip out and not aspirated at any stage, no matter how slow the drainage. Alternative methods of CSF removal include temporary or continuous drainage. The latter entails a high risk of secondary infection and makes nursing of the infant difficult. Temporary drainage may offer a more physiological alternative to tapping without increasing the risk of infection.

1 Marlin AE. Protection of the cortical mantle in premature infants with posthaemorrhagic hydrocephalus. Neurosurgery 1980;7:464-8.

2 Hislop JE, Dubowitz LMS, Kaiser AM, Singh MP, Whitelaw AGC. Outcome of infants shunted for post haemorrhagic ventricular dilatation. Dev Med Child Neurol 1988;30:451-6.

3 Gaskill SJ, Marlin AE, Rivera S. The subcutaneous ventricular reservoir: an effective treatment for posthaemorrhagic cular reservoir: an effective treatment for postha

4 Leonhardt A, Steiner H-H, Linderkamp O. Management of posthaemorrhagic hydrocephalus with a subcutaneous ventricular catheter reservoir in premature infants. Arch Dis Child 1989;64:24-8. 